\title{
Clinical characteristics and surgical treatment of spinal metastases from pancreatic cancer: a single-center retrospective study
}

\author{
Shuzhong Liu ${ }^{1 \#}$, Xi Zhou ${ }^{1 \#}$, An Song ${ }^{2}$, Zhen Huo ${ }^{3}$, Yipeng Wang ${ }^{1}$, Yong Liu ${ }^{1}$ \\ ${ }^{1}$ Department of Orthopaedic Surgery, Peking Union Medical College Hospital, Peking Union Medical College and Chinese Academy of Medical \\ Sciences, Beijing, China; ${ }^{2}$ Department of Endocrinology, Key Laboratory of Endocrinology, National Health and Family Planning Commission, \\ Peking Union Medical College Hospital, Chinese Academy of Medical Science \& Peking Union Medical College, Beijing, China; ${ }^{3}$ Department of \\ Pathology, Peking Union Medical College Hospital, Chinese Academy of Medical Science \& Peking Union Medical College, Beijing, China \\ Contributions: (I) Conception and design: S Liu, X Zhou, Y Liu; (II) Administrative support: S Liu, X Zhou, Y Liu; (III) Provision of study materials \\ or patients: Y Liu; (IV) Collection and assembly of data: S Liu, X Zhou, A Song, Z Huo; (V) Data analysis and interpretation: S Liu, X Zhou, Y \\ Wang, Y Liu; (VI) Manuscript writing: All authors; (VII) Final approval of manuscript: All authors. \\ "These authors contributed equally to this work. \\ Correspondence to: Yong Liu. Department of Orthopaedic Surgery, Peking Union Medical College Hospital, Peking Union Medical College and \\ Chinese Academy of Medical Sciences, No. 1 Shuaifuyuan Wangfujing, Beijing, China. Email: liuyong_pumch@163.com.
}

Background: To improve the understanding of the characteristics of rare pancreatic cancer spinal metastatic disease and share our experience in coping with this disease. Although spinal metastasis of pancreatic cancer is extremely rare clinically, and the prognosis of the primary tumor is very poor, pancreatic cancer spinal metastasis has received insufficient attention in clinical practice and is only described in a limited number of case reports or series. The purpose of the present study is to discuss the clinical features, prognostic characteristics, and treatment of individuals with pancreatic cancer spinal metastases.

Methods: Four patients with clinical symptoms caused by metastatic spinal pancreatic cancer (MSPC) were selected from patients treated in our department between January 2010 and January 2020. Patients' clinical and surgical records, imaging data, and pathology reports were reviewed by our team. A retrospective analysis of patient clinical data was conducted.

Results: Of the four patients, one was male and three were female. The average age was 68.0 (range, 61-79) years old. The average time between the pancreatic cancer diagnosis and the diagnosis of spinal metastases was 10.5 (range, 0-24) months. Spinal metastatic disease was primarily found in the thoracic spine $(\mathrm{n}=3 ; 75.0 \%)$, and the lumbar spine $(\mathrm{n}=2 ; 50.0 \%)$. During follow-up, local tumor progression was found in all four patients (100\%), all of whom died of pancreatic cancer during follow-up visits. The median time between spinal surgery and death was 16.3 (range, 12-19) months.

Conclusions: Taken together, pancreatic cancer patient that have spinal metastases exhibit a poor prognosis, with a survival time shorter than for any other malignant tumor. Percutaneous vertebroplasty may become an effective treatment option for pancreatic cancer spinal metastasis, which can significantly improve the patient's symptoms.

Keywords: Pancreatic cancer; spinal metastasis; surgical treatment; percutaneous vertebroplasty; adjuvant therapy; prognosis

Submitted May 25, 2020. Accepted for publication Sep 05, 2020.

doi: 10.21037/apm-20-1168

View this article at: http://dx.doi.org/10.21037/apm-20-1168 


\section{Introduction}

Pancreatic cancer is a common malignant tumor. It has the worst prognosis of all digestive system tumors and is known as "the king of cancer" (1-3). Pancreatic cancer accounts for about $1-2 \%$ of malignant tumors in the whole body (1-3). This catastrophic disease usually has an insidious onset, rapid progression, a short course of disease, and lacks typical clinical manifestations in the early stage. Almost all cases are diagnosed when local progression or distant metastasis occurs $(4,5)$. Because of this, and because of the deep location of the tumor, surrounded by anatomically complex tissues and organs, the opportunity for successful surgery is often lost when visiting the doctor. At present, due to technical improvements in diagnosis and treatment, the survival time of patients has been prolonged, and cases with metastatic spinal pancreatic cancer (MSPC) have increased, now accounting for about $7.3 \%$ of pancreatic cancer patients $(5,6)$.

Few studies to date have reported on spinal metastases from pancreatic cancer, only seen in some case reports or small-scale case series studies (7-9). In the present retrospective study, we assessed data pertaining to four cases of MSPC that received spinal surgical intervention in a single center over 10 years. Among over 1,000 individuals with spinal metastases treated by the bone tumor center of Peking Union Medical College Hospital in the past decade, only four patients were diagnosed with pancreatic cancer spinal metastases. We report on these four MSPC patients and discuss strategies for assessing clinical symptoms, diagnosis, treatment, prolonging survival in advanced cases, and improving the quality of life of these patients. We present the following article in accordance with the STROBE reporting checklist (available at http://dx.doi. org/10.21037/apm-20-1168).

\section{Methods}

Four consecutive patients with spinal metastases originating from pancreatic cancer were selected from patients surgically treated in our department from January 2010 January 2020. Diagnoses in these patients were confirmed through postoperative pathological analyses. We reviewed all imaging data, pathological reports, clinical data, and surgical records pertaining to these patients confirmed the final diagnosis. The clinical data and surgical records, imaging features, and pathological reports of all these patients were reviewed. The final follow-up was completed in January 2020. The study was conducted in accordance with the Declaration of Helsinki (as revised in 2013). The hospital ethics committee (No. S-K1268) approved the present study, and the informed consent for the surgical procedure of all participants was obtained.

All patients were evaluated by radiography, spine CT, and MRI. Systemic metastases in these patients were evaluated via bone scans, PET-CT, chest, and abdomen CT scans were performed to assess systemic metastases. Tumor marker levels in the peripheral blood were analyzed in all patients. Frankel scores and ASIA injury classification criteria were utilized as a means of assessing the preoperative neurological status in all analyzed patients, while VAS, ECOG, and Karnofsky scores were employed to gauge quality of life. Surgery performed via a standard method is conducted by our surgical team led by Professor Liu (10). Spinal stability was evaluated based upon the SINS system, while revised Tokuhashi and Tomita scoring was used to evaluate patient prognosis and to plan surgical approaches as appropriate.

Imaging assessments (X-ray, CT or MRI) of primary lesions and spinal metastatic lesions were conducted three and six months postoperatively, and at six-month intervals for the following two years. Follow-up data was collected via telephone interviews and through in-person outpatient follow-up. At three months post-surgery, quality of live was evaluated using VAS and ECOG scores.

\section{Statistical analysis}

OS was the time from spinal surgery to the death of the patient or the end of January 2020. PFS was the time from the date of spinal surgery to local disease progression after surgery (based on radiological assessment). Statistical data were analyzed using SPSS 23.0. Counting data were described by proportion and frequency.

\section{Results}

\section{Clinical characteristics}

Of the four patients, one was male and three were female. The average age was 68.0 (range, 61-79) years. Two patients had undergone resection of the primary pancreatic cancer before surgery, and the other two patients had not undergone surgical treatment of the primary tumor. The average time between the pancreatic cancer resection and the diagnosis of spinal metastases was 10.5 (range, 


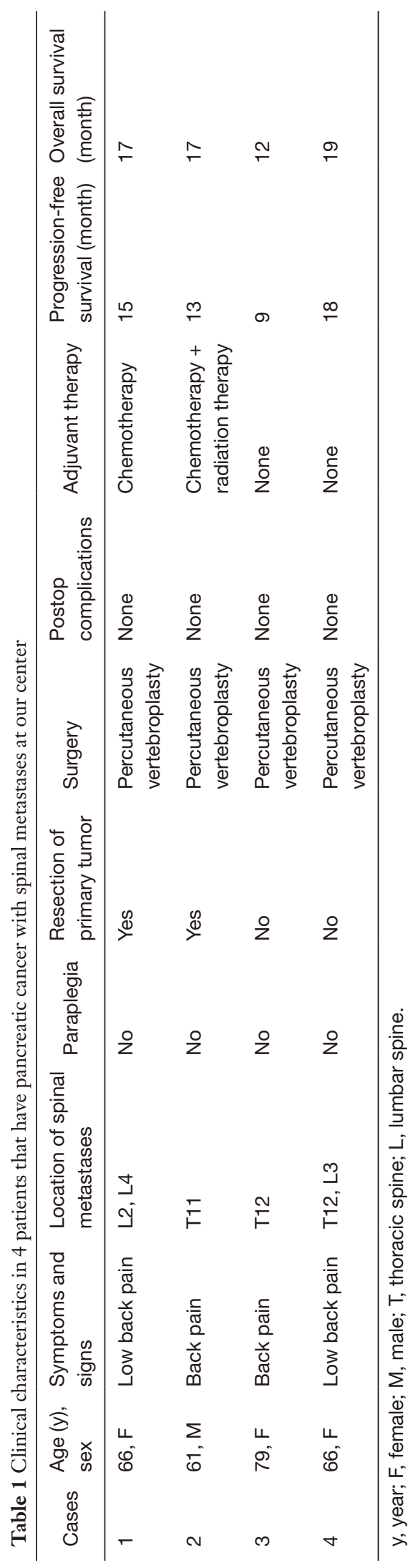

0-24) months. None of the four patients had bone or visceral metastases on admission. Two patients received chemotherapy while the other two patients refused further adjuvant therapy after due consideration.

The clinical data of the four MSPC patients are compiled in Table 1. All patients had progressive low back pain before surgery. On average preoperative symptoms were present for 2.0 (range, 1-3) months. None of the four patients had paralysis of the lower extremities or bowel and bladder disturbances. The locations of spinal metastasis revealed three instances of metastases in the thoracic spine and two instances of lumbar spine metastases.

The imaging features were quite different from those seen in other spinal metastatic tumors. Of the four patients with MSPC, two had spinal metastases with osteogenic changes, and the other two patients had spinal metastases with osteolytic changes. Osteogenic or osteolytic lesions can be detected on X-rays and CT scans, and spinal metastases can be accompanied by paravertebral soft-tissue masses. MRI showed iso-hypointensity on T1-weighted images and iso-hyperintensity on T2-weighted images (Figure 1). Enhanced MRI showed that the tumor was significantly enhanced, similar to other spinal metastatic lesions. Common radiological findings in this cohort are non-specific, and compression fractures are not common $(\mathrm{n}=2 ; 50.0 \%)$. The characteristics of these four patients and related scoring data are shown in Table 2.

Surgical treatment was done by our departmental team led by Professor Liu. All physicians on this team had received professional training for at least 5 years. All patients underwent percutaneous vertebroplasty with bone cement augmentation technology. Average intraoperative blood loss was 30 (range, 20-50) mL. Postoperative pathological examination confirmed the pancreatic cancer spinal metastasis diagnosis. Two patients continued their original treatment plan after spinal surgery. All four patients received postoperative bisphosphonate treatment.

\section{Follow-up}

In this retrospective study, no perioperative complications were observed. Postoperatively, all patients' local pain was ameliorated. During the three-month follow-up, the state of the neurological system showed a Frankel score improvement of 1-2 grades. During follow-up, all four patients exhibited local tumor progression $(100 \%)$ and the progression-free survival was 13.8 (range, 9-18) months. During follow-up, all four patients died from this disease 

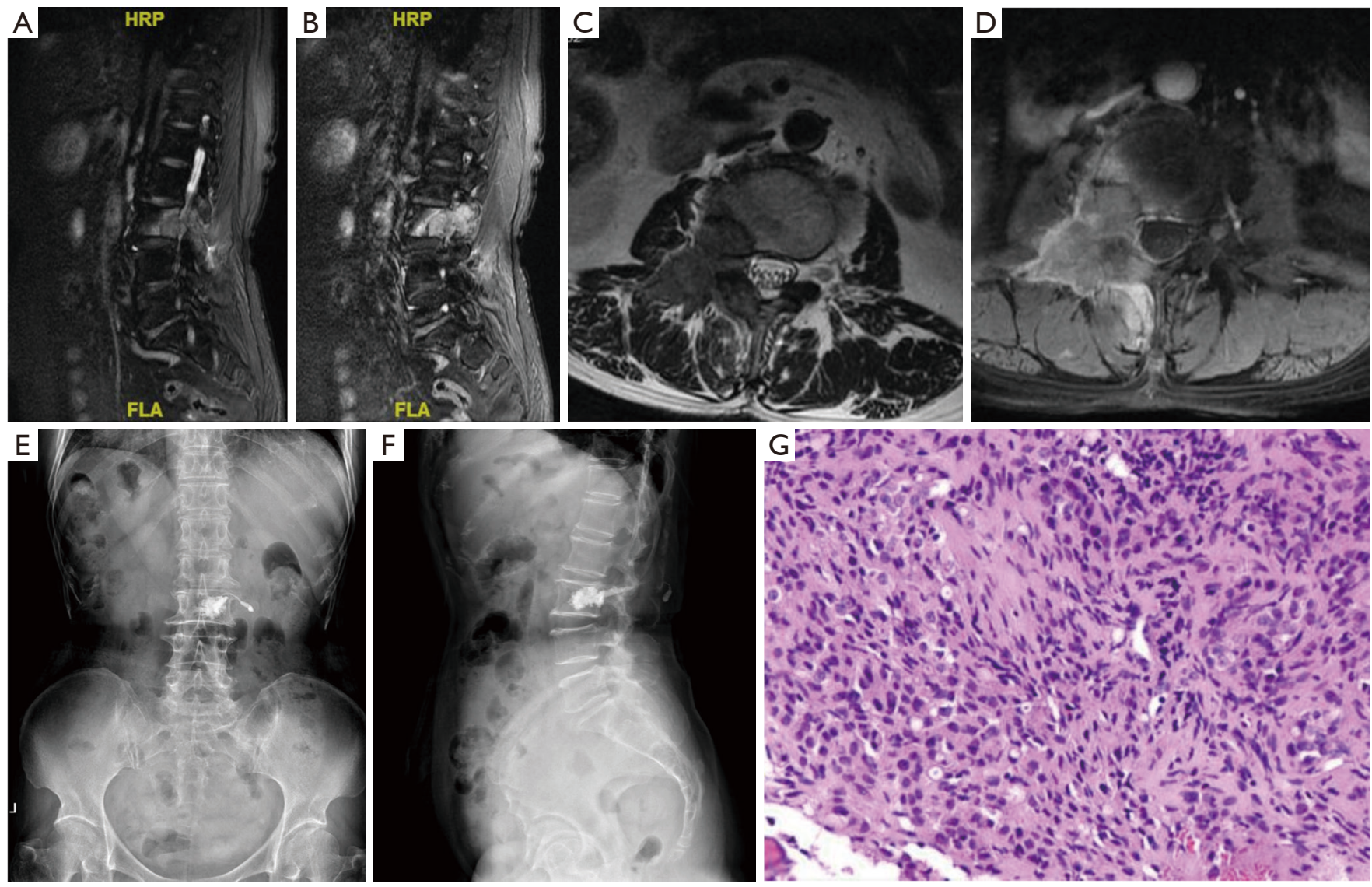

Figure 1 Representative radiographic and pathological images from a 66-year-old female patient (case 4). (A,B,C,D) Preoperative sagittal and transverse MRI scans showing L3 vertebral metastases. (E,F) Postoperative lumbar spine X-ray images. (G) Microphotography exhibiting characteristic tumor cell nests. H\&E, original magnification $100 \times$.

with an average overall survival time was 16.3 (range, $12-$ 19) months.

\section{Discussion}

Pancreatic cancer is known as "the king of cancer", with a low complete resection rate, short survival time, high mortality rate, and extremely poor prognosis (1-3). For patients with pancreatic cancer, treatment after spinal metastasis is particularly important to improve the quality of life $(9,11,12)$. Because of the poor prognosis of the primary tumor, MSPC has not received sufficient attention in clinical practice and has been described in a limited number of case reports or case series (7-9). There is a lack of clinical treatment experience for this situation, and there is no consensus on the treatment strategy.

Lower back pain is a frequent symptom of MSPC.
However, the pain lacks specificity and symptoms are often hidden, which can easily lead to missed diagnosis and misdiagnosis $(7-9,13,14)$. Consistent with other spinal metastasis types, the location of the spinal cord injury in MSPC cases dictates the resultant neurological deficits (7-9). Cervical spinal cord compression often manifests as upper limb dysfunction, dizziness, and weakness. Patients with lesions in the thoracolumbar spine might show symptoms of low back pain, abnormal sensation, and weakness of the lower extremities. In our study, all patients had progressive low back pain before surgery. The average preoperative symptom duration was 2.0 (range, 1-3) months.

Imaging examination is important for the early diagnosis of MSPC. The X-ray or CT of MSPC may show osteogenic or osteolytic bone destruction, which is consistent with the results of our study (7-9). It is easy to miss the diagnosis with early $\mathrm{X}$-ray plain films. CT 


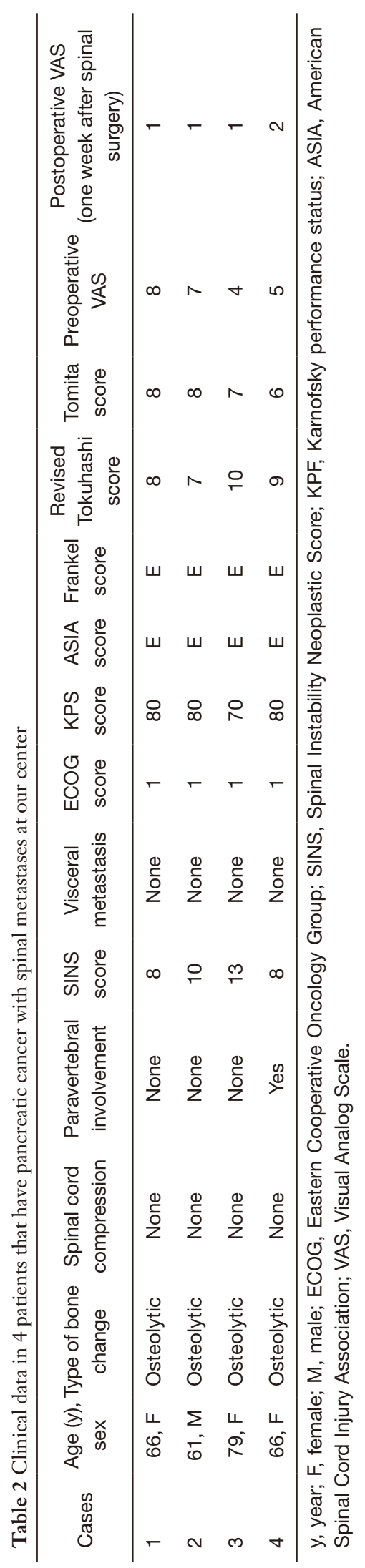

and MRI are more sensitive, and can be comprehensively evaluated for the location, degree, and integrity of the spinal structure and spinal nerve compression caused by the metastatic tumor (15-17). Bone scan is very important for the diagnosis of bone metastasis and makes missed diagnosis much less likely. The lesion can be found earlier and more accurately, and multiple suspected bone metastases can be found from a single examination. Overexpression of glucose transporter proteins (Glut1-4) on the surface of pancreatic cancer cells is positively correlated with the degree of glycolysis enhancement in cancer cells, which is the theoretical basis for the application of ${ }^{18} \mathrm{~F}-\mathrm{FDG}$ PET/ CT in the diagnosis of pancreatic cancer and metastatic lesions $(18,19)$. The specificity of PET/CT for detecting pancreatic cancer is $64-100 \%$, sensitivity is reported to be $71-100 \%$, and accuracy is $83-93 \%(18,19)$. The latest high-resolution PET/CT can measure pancreatic cancer with a diameter of $7 \mathrm{~mm} .{ }^{18} \mathrm{~F}$-FDG PET/CT has a higher diagnostic efficiency in pancreatic cancer and its metastatic tumors. PET/CT whole-body imaging is helpful in finding the distant metastases of pancreatic cancer, such as bone and lung, which cannot be easily found by other methods $(18,19)$. Nishiyama reported 42 cases of pancreatic cancer of which 16 cases of bone metastases were clinically diagnosed, and a further 23 cases of bone metastases were clinically diagnosed by PET/CT in the remaining 26 cases (20).

With the evolution of diagnosis and treatment methods, surgery is one of the comprehensive treatment measures for spinal metastases, and its key role has been widely recognized and clinically confirmed (7-9). The purpose of surgical treatment for MSPC is to relieve pain, reduce and prevent damage to the neurological system, ensure immediate or stable improvement in spinal stability, and maximize patient's quality of life (7-9,21). These surgical indications must be strictly mastered: (I) progressive neurological dysfunction or other severe symptoms; (II) intractable pain that fails to respond to any form of conservative treatment; (III) confirmation of the diagnosis by pathology; (IV) radio- and chemoresistant tumors; (V) instability of the spine or collapse of the vertebral body, with or without neurological dysfunction; (VI) patients with an expected survival period of greater than three months. Based on other previously published case reports and case series, we believe that although the prognosis of patients with MSPC is relatively poor, the effect of spinal surgery is significant. Moreover, there is no significant difference in terms of surgical efficacy and prognosis between open surgery and percutaneous vertebroplasty. The purpose of 
our surgical intervention in MSPC patients was to reduce symptoms, alleviate any compression of the spinal cord and associated nerve roots, and to enhance the stability of the spine. When staging patients based upon revised Tokuhashi and Tomita scores, many factors including numbers of spinal metastases and visceral metastases should be considered, although they are not the ultimate determinants of any operations that are ultimately conducted $(22,23)$. In patients exhibiting multiple spinal metastases, we are more inclined to solve the most important problem at that time, in line with the surgical indications.

There is no international consensus on whether to choose open radical surgery or percutaneous vertebroplasty for spinal metastases (7-9). Percutaneous vertebroplasty has a low risk and has some effect on relieving pain symptoms. Radical surgery can achieve better local control of the tumor and may, if combined with comprehensive treatment, offer the possibility of a cure, although with high risk and difficulty. Reconstruction of spinal stability is also more complicated. For patients with single, slower primary tumor growth (such as thyroid cancer, kidney cancer, and breast cancer) spinal metastases or patients with severe spinal cord compression and paraplegia, active radical surgery is recommended, combined with comprehensive treatment, which can achieve a more satisfactory result. As MSPC has a poor prognosis, the primary surgical method in this series is percutaneous vertebroplasty (7-9). In this study, no clear perioperative complications were observed, and postoperatively, local pain improved in all patients. During the three-month follow-up, the neurological conditions showed a Frankel score improvement of 1-2 grades. The patients' PFS and OS were also satisfactory during the follow-up process. The application of minimally invasive percutaneous vertebroplasty using bone cement in the treatment of MSPC patients offers advantages including: (I) bone cement can be administered using local anesthesia, thereby decreasing surgical risk under local anesthesia to minimize the risk of surgery; (II) the surgical damage is less, and the effects of treatment are significant; (III) the scope of application of bone cement for MSPC also has the potential to be expanded not only to reinforce spinal metastases but also to treat other affected bones; (IV) for patients with suspected MSPC, tissue biopsy should be performed to confirm the pathological diagnosis at the same time; (V) bone cement materials have the potential to inhibit bacterial growth and tumor formation. The new bone cement materials can be loaded with certain chemotherapy and targeted therapy drugs, which is expected to further control local lesions. Therefore, percutaneous vertebroplasty provides a new way of thinking and options for the early diagnosis and reasonable treatment of patients with MSPC.

Non-surgical treatments for bone metastases from pancreatic cancer include targeted therapy, bisphosphonates, radiotherapy, and chemotherapy (24-28). Bisphosphonate is an important treatment for most patients with spinal metastases (28). It effectively decreases the risk of fractures in the spine, pelvis, and other extraspinal bones. In our study, all four patients received bisphosphonate therapy after surgery to further prevent spinal and systemic skeletalrelated complications.

It is generally believed that the survival of individuals with spinal metastases is closely linked to the biological behavior of the primary tumor, treatment methods, and the patient's overall condition (7-9,24-27). Because pancreatic cancer is highly malignant and difficult to treat, the prognosis for MSPC is extremely poor. To obtain a longer survival period and better quality of life for such patients, active multimodal treatment should still be carried out, and full attention should be paid to protect their immune function, supplemented by biological immunotherapy and symptomatic treatment as appropriate $(29,30)$. Therefore, it is important to be able to estimate the survival prognosis of each patient to facilitate the formulation and implementation of a comprehensive treatment plan.

Spinal metastases of unknown primary (SMUP) are challenging in the treatment of patients with malignant spinal metastases, and the concepts of systematics and translational medicine are crucial in clinical and scientific research practices (31). Over the past few years, managing these patients has partially become easier due to a better understanding of the biological characteristics of SMUP (31). The improvements in knowledge facilitated clinical decision-making by integrating genetic and molecular characteristics of SMUP into a multi-step diagnostic process. In this regard, Argentiero and coworkers postulated that a pragmatic investigation plan and therapy for SMUP could not follow a single template (31). The treatment must reflect different pathophysiological dynamics and should be based on systematic methodology and high-quality data derived from clinical trials (31). However, there are only a few reports combining basic research and clinical aspects of MSPC. Therefore, it is necessary to encourage the enrollment of patients in clinical trials, including their genetic and molecular characterization, and to combine standard chemotherapy utilizing treatment by bone-modifying agents with 
molecular-targeted drugs.

MSPC is a clinical challenge with unexplained biological characteristics (32). The systemic treatment of MSPC would be an exciting and promising research topic. Recently, the combination of nitrogen-containing bisphosphonates (e.g., zoledronic acid) and nab-paclitaxel has been demonstrated to reduce fibrosis, peritoneal dissemination, angiogenesis, and cell proliferation (33). In addition, preclinical studies have shown that zoledronic acid exerts an antitumor activity toward pancreatic ductal adenocarcinoma (PDAC) cells (33). Moreover, zoledronic acid stimulates the antitumor response of lymphocytes by activating $\gamma \delta$-type $\mathrm{T}$ cell receptors, improving the survival rate of the first bonerelated events (34). It is worth noting that zoledronic acid appears to modulate innate immunity by acting on tumor-associated macrophages. In addition, Argentiero and coworkers documented that the new immunotherapy strategy targeting the Wnt pathway can enhance the cytotoxicity of pancreatic cancer cells and restore antitumor immunity in lymph node-positive patients (35). Tumorstromal interactions are important for the progression of PDAC, and mast cells play a crucial role in the resistance to gemcitabine/paclitaxel treatment (36,37). High levels of proinflammatory/immunosuppressive cytokines in PDAC patients are associated with the absence of response to chemotherapy (37). In principle, multi-modality therapy of pancreatic cancer bone metastasis implies that interactions between different treatment modes will likely be present.

In the current study, two of the four patients received throughout the course of the disease only percutaneous vertebroplasty. Pancreatic tumor resection or adjuvant therapy was not performed due to patients' wishes and the clinical stage of the tumor. However, of all four patients, patient 4 had the longest survival time, 19 months, which showed that the treatment mode of MSPC could be significantly improved and optimized. The improvement of the effects of systemic treatment is likely closely related to the individual differences in the pathology, molecular genetics, and types of mutations among MSPC patients. This result also gives clinical researchers important hints to pay attention to individual differences and overall cooperation in treatment.

There are multiple limitations to the present analysis. For one, this was a retrospective study and the sample size was small. It was not possible to conduct more systematic and comprehensive statistical analyses, and the specific prognostic factors associated with MSPC cannot be determined. Secondly, this study only included patients with MSPC undergoing spinal surgery and did not include patients who did not receive spinal surgery. This will bring some selective bias to the results of the study. Since this was a retrospective study spanning ten years, we could not study the molecular characteristics of tumors at diagnosis and during the treatment. Moreover, during the past ten years, the rapid development of targeted molecular therapies for bone metastasis of pancreatic cancer took place, representing another limitation of this study. Even so, this study is the only single-center retrospective study in Asia that discusses the surgical treatment of MSPC. The study involved over 1,000 spinal metastasis patients over a 10-year period at the Peking Union Medical College Hospital. The surgical and clinical data, therefore, have significance for clinical guidance.

\section{Conclusions}

In conclusion, although patients with pancreatic cancer spinal metastases exhibit poor prognosis, surgical treatment can improve the patient's quality of life. For patients with MSPC without spinal cord compression, we recommend percutaneous vertebroplasty to effectively control local spinal lesions and relieve clinical symptoms. In those that have uncontrolled pain or neurological deficits, open tumor resection, spinal cord decompression, and internal fixation surgery can be considered. By better grasping the clinical features of MSPC, it is helpful to formulate clinical treatment plans to enhance the quality of life of patients and improve their prognosis.

\section{Acknowledgments}

We would like to thank our colleagues at the Departments of General surgery, Anesthesiology, Pathology, Nuclear medicine, Radiology, and Orthopaedic surgery for their support.

Funding: This study was supported by Peking Union Medical College Graduate Student Innovation Fund (2018) (Project No. 2018-1002-02-08; Grant recipient: SL).

\section{Footnote}

Reporting Checklist: The authors have completed the STROBE reporting checklist. Available at http://dx.doi. org/10.21037/apm-20-1168

Data Sharing Statement: Available at http://dx.doi. 
org/10.21037/apm-20-1168

Conflicts of Interest: All authors have completed the ICMJE uniform disclosure form (available at http://dx.doi. org/10.21037/apm-20-1168). The authors have no conflicts of interest to declare.

Ethical Statement: The authors are accountable for all aspects of the work in ensuring that questions related to the accuracy or integrity of any part of the work are appropriately investigated and resolved. The study was conducted in accordance with the Declaration of Helsinki (as revised in 2013). The study was approved by institutional ethics board of Peking Union Medical College Hospital (No. S-K1268) the registration number of ethics board) and individual consent for this retrospective analysis was waived.

Open Access Statement: This is an Open Access article distributed in accordance with the Creative Commons Attribution-NonCommercial-NoDerivs 4.0 International License (CC BY-NC-ND 4.0), which permits the noncommercial replication and distribution of the article with the strict proviso that no changes or edits are made and the original work is properly cited (including links to both the formal publication through the relevant DOI and the license). See: https://creativecommons.org/licenses/by-nc-nd/4.0/.

\section{References}

1. Li D, Morris JS, Liu J, et al. Body Mass Index and Risk, Age of Onset, and Survival in Patients With Pancreatic Cancer. JAMA 2009;301:2553-62.

2. Siegel RL, Miller KD, Jemal A. Cancer statistics, 2018. CA Cancer J Clin 2018;68:7-30.

3. Chen $\mathrm{W}$, Zheng R, Baade PD, et al. Cancer statistics in China, 2015. CA Cancer J Clin 2016;66:115-32.

4. Conroy T, Desseigne F, Ychou M, et al. FFOLFIRINOX Versus Gemcitabine for Metastatic Pancreatic Cancer. N Engl J Med 2011;364:1817-25.

5. Chiang KC, Yu CC, Chen JR, et al. Oncocytic-type intraductal papillary mucinous neoplasm (IPMN)-derived invasive oncocytic pancreatic carcinoma with brain metastasis: a case report. World J Surg Oncol 2012;10:138.

6. Iguchi $\mathrm{H}$, Yasuda M, Matsuo T, et al. Clinical features and management of pancreatic cancer with bone metastases. Nippon Shokakibyo Gakkai Zasshi 2004;101:872-8.

7. Kim DH, Kim DH, Kim HS, et al. Delayed Epidural Mucin Collection After Surgery for Spinal Metastatic
Pancreatic Adenocarcinoma. Korean J Spine 2017;14:11-3.

8. Chih YP, Wu WT, Lin CL, et al. Vertebral Compression Fracture Related to Pancreatic Cancer With Osteoblastic Metastasis: A Case Report and Literature Review. Medicine (Baltimore) 2016;95:e2670.

9. Rades D, Huttenlocher S, Schild SE, et al. Metastatic Spinal Cord Compression From Pancreatic Cancer. Anticancer Res 2014;34:3727-30.

10. Liu S, Zhou X, Song A, et al. Successful treatment of malignant thymoma with sacrum metastases: A case report and review of literature. Medicine (Baltimore) 2018;97:e13796.

11. Pneumaticos SG, Savidou C, Korres DS, et al. Pancreatic cancer's initial presentation: back pain due to osteoblastic bone metastasis. Eur J Cancer Care (Engl) 2010;19:137-40.

12. Borad MJ, Saadati H, Lakshmipathy A, et al. Skeletal metastases in pancreatic cancer: a retrospective study and review of the literature. Yale J Biol Med 2009;82:1-6.

13. Takuma Y, Kawai D, Makino Y, et al. Vertebral metastasis of intraductal papillary mucinous tumor of the pancreas. Pancreas 2006;33:206-8.

14. Joffe N, Antonioli DA. Osteoblastic bone metastases secondary to adenocarcinoma of the pancreas. Clin Radiol 1978;29:41-6.

15. Parikh SN, Grawford AH. Orthopaedic implications in the management of pediatric vertebral and spinal cord tumors: a retrospective review. Spine 2003;28:2390-6.

16. Trumm CG, Pahl A, Helmberger TK, et al. CT fluoroscopy-guided percutaneous vertebroplasty in spinal malignancy: technical results, PMMA leakages, and complications in 202 patients. Skeletal Radiol 2012;41:1391-400.

17. Tirkes T, Sandrasegaran K, Sanyal R, et al. Secretinenhanced MR cholangiopancreatography: spectrum of findings. Radiographics 2013;33:1889-906.

18. Higashi T, Saga T, Nakamoto Y, et al. Diagnosis of Pancreatic Cancer Using fluorine-18 Fluorodeoxyglucose Positron Emission Tomography (FDG PET) --Usefulness and Limitations in "Clinical Reality". Ann Nucl Med 2003;17:261-79.

19. Xu HX, Chen T, Wang WQ, et al. Metabolic Tumour Burden Assessed by 18F-FDG PET/CT Associated With Serum CA19-9 Predicts Pancreatic Cancer Outcome After Resection. Eur J Nucl Med Mol Imaging 2014;41:1093-102.

20. Nishiyama Y, Yamamoto Y, Yokoe K, et al. Contribution of whole body FDG-PET to the detection of distant matastasis in pancreatic cancer. Ann Nucl Med 
2005;19:491-7.

21. Connor AA, Denroche RE, Jang GH, et al. Association of distinct mutational signatures with correlates of increased immune activity in pancreatic ductal adenocarcinoma. JAMA Oncol 2017;3:774-83.

22. Tokuhashi $\mathrm{Y}$, Matsuzaki H, Oda H, et al. A revised scoring system for preoperative evaluation of metastatic spine tumor prognosis. Spine (Phila Pa 1976) 2005;30:2186-91.

23. Tomita K, Kawahara N, Kobayashi T, et al. Surgical strategy for spinal metastases. Spine (Phila Pa 1976) 2001;26:298-306.

24. Uesaka K, Boku N, Fukutomi A, et al. Adjuvant Chemotherapy of S-1 Versus Gemcitabine for Resected Pancreatic Cancer: A Phase 3, Open-Label, Randomised, Non-Inferiority Trial (JASPAC 01). Lancet 2016;388:248-57.

25. Chantrill LA, Nagrial AM, Watson C, et al. Precision medicine for advanced pancreas cancer: The individualized molecular pancreatic cancer therapy (IMPaCT) trial. Clin Cancer Res 2015;21:2029-37.

26. Vincent A, Herman J, Schulick R, et al. Pancreatic cancer. Lancet 2011;378:607-20.

27. Lunardi S, Muschel RJ, Brunner TB. The stromal compartments in pancreatic cancer: are there any therapeutic targets? Cancer Lett 2014;343:147-55.

28. Bhowmik D, Song X, Intorcia M, et al. Examination of burden of skeletal-related events in patients naive to denosumab and intravenous bisphosphonate therapy in bone metastases from solid tumors population. Curr Med Res Opin 2019;35:513-23.

29. Sideras K, Braat H, Kwekkeboom J, et al. Role of the immune system in pancreatic cancer progression and immune modulating treatment strategies. Cancer Treat

Cite this article as: Liu S, Zhou X, Song A, Huo Z, Wang Y, Liu Y. Clinical characteristics and surgical treatment of spinal metastases from pancreatic cancer: a single-center retrospective study. Ann Palliat Med 2021;10(2):1276-1284. doi: 10.21037/apm20-1168
Rev 2014;40:513-22.

30. Sahin IH, Askan G, Hu ZI, et al. Immunotherapy in pancreatic ductal adenocarcinoma: An emerging entity? Ann Oncol 2017;28:2950-61.

31. Argentiero A, Solimando AG, Brunetti O, et al. Skeletal Metastases of Unknown Primary: Biological Landscape and Clinical Overview. Cancers (Basel) 2019;11:1270.

32. Argentiero A, Calabrese A, Solimando AG, et al. Bone metastasis as primary presentation of pancreatic ductal adenocarcinoma: A case report and literature review. Clin Case Rep 2019;7:1972-6.

33. Gonzalez-Villasana V, Rodriguez-Aguayo C, Arumugam T, et al. Bisphosphonates inhibit stellate cell activity and enhance antitumor effects of nanoparticle albumin-bound paclitaxel in pancreatic ductal adenocarcinoma. Mol Cancer Ther 2014;13:2583-94.

34. Oberg HH, Wesch D, Kalyan S, et al. Regulatory Interactions Between Neutrophils, Tumor Cells and T Cells. Front Immunol 2019;10:1690.

35. Argentiero A, De Summa S, Di Fonte R, et al. Gene Expression Comparison between the Lymph NodePositive and -Negative Reveals a Peculiar Immune Microenvironment Signature and a Theranostic Role for WNT Targeting in Pancreatic Ductal Adenocarcinoma: A Pilot Study. Cancers (Basel) 2019;11:942.

36. Heinemann V, Reni M, Ychou M, et al. Tumour-stroma interactions in pancreatic ductal adenocarcinoma: rationale and current evidence for new therapeutic strategies. Cancer Treat Rev 2014;40:118-28.

37. Porcelli L, Iacobazzi RM, Di Fonte R, et al. CAFs and TGF-beta Signaling Activation by Mast Cells Contribute to Resistance to Gemcitabine/Nabpaclitaxel in Pancreatic Cancer. Cancers (Basel) 2019;11:330. 